GP227 CLINICAL SPECTRUM OF CLASSICAL GALACTOSAEMIA ASSOCIATED WITH FRIEDREICH'S ATAXIA IN A PAEDIATRIC COHORT IN THE REPUBLIC OF IRELAND AN UPDATE

${ }^{1}$ Ritma Boruah*, Siobhan Siobhan ${ }^{2}, 2$ Orla Franklin, ${ }^{3}$ Declan O' Rourke, ${ }^{3}$ Bryan Lynch, ${ }^{4}$ Sally Ann Lynch, 'Joanne Hughes, 'Ahmad A Monavari, ${ }^{1}$ Ellen Crushell, ${ }^{1}$ Ina Knerr. 'National Centre for Inherited Metabolic Disorders, Temple Street Childrens University Hospital, Dublin, Ireland; ${ }^{2}$ National Centre for Paediatric Cardiology, Our Lady's Children's Hospital, Crumlin, Dublin, Ireland; ${ }^{3}$ Department of Neurology, Temple Street Children's University Hospital, Dublin, Ireland; ${ }^{4}$ Department of Clinical Genetics, Our Lady's Children's Hospital Crumlin, Dublin, Ireland

10.1136/archdischild-2019-epa.286

Despite early diagnosis through Newborn Bloodspot Screening and strict dietary treatment, there are long-term complications of Classical Galactosaemia, including female infertility, osteopenia, and, in some cases, learning disabilities or neurological symptoms such as tremor or ataxia. Therefore, neurological symptoms may easily be attributed to an underlying diagnosis of Classical Galactosaemia. However, the coexistence of classical Galactosaemia and Friedreich's Ataxia (FRDA) was previously reported amongst the Irish Traveller population. Classical Galactosaemia and FRDA are both autosomal recessive conditions, the gene loci for which are located on either side of the centromere of chromosome 9. FRDA is one of the most common forms of autosomal-recessive ataxia and slowly progressive; it also commonly involves the heart. We here present the clinical spectrum of 10 Irish patients currently being treated in our Metabolic Centre in whom a diagnosis of Classical Galactosaemia together with FRDA was made. All patients were diagnosed with Classical Galactosaemia through Newborn Screening first. All were diagnosed with the common Classical Galactosaemia GALT mutation Q188R/Q188R. Eight of the ten patients later presented with progressive ataxia, between the ages of 7-14 years. One child presented in cardiac failure secondary to dilated cardiomyopathy at 7 years of age. It was noted that he was not ataxic at presentation of FRDA and that he had normal tendon reflexes. Another patient who was diagnosed at 6 years of age still had an essentially normal neurological exam almost 4 years later. The diagnosis of FRDA was confirmed by detecting the common pathogenic GAA repeat expansion mutations in both alleles of the frataxin gene (FXN) in affected individuals.

Taken together, neurological signs may easily be attributed to an established diagnosis of Classical Galactosaemia. However, one should be vigilant for the coexistence of Classical Galactosaemia and FRDA in a high-risk population, particularly when monitoring patients with a complex phenotype, such as a neurological or cardiac presentation.

\section{GP228 NEUROPSYCHOLOGICAL PROFILE ON NEPSY IN CHILDREN AT PERINATAL RISK WITH AND WITHOUT CEREBRAL PALSY - SIMILAR OR DIFFERENT?}

Snjezana Bilac*, Tatjana Puljiz, Lidija Sajert, Katarina Bosnjak Nad. Special Hospital for Children with Neurodevelopmental and Motor Disorders, Zagreb, Croatia

\subsection{6/archdischild-2019-epa.287}

Objective To examine the neuropsychological profile of children at perinatal risk with or without $\mathrm{CP}$, compare them to each other and to the normative group. It is expected that more specific cognitive deficits will be found in both clinical groups in relation to the normative group, as well as more deficits in children with $\mathrm{CP}$ compared to children at perinatal risk without $\mathrm{CP}$.

Design/study groups clinical sample of 46 children with milder CP (the CP group) and 55 children at perinatal risk without CP (the group at risk) in the age range between 5 and 12 years with normal verbal intelligence (according to WISC-III) were examined. The groups are equalized in terms of demographic variables (child's age and sex; mother's education) as well as in terms of perinatal variables (gestational age, birth weight, Apgar scores, presence of brain lesion $78 \%$ in each group), and EEG findings at the time of examination.

Main outcome measures Developmental Neuropsychological Assessment (NEPSY) has been applied to measure neuropsychological functions, all the core subtests except Phonological Processing.

Results Both clinical groups had significantly lower results in 12 out of 13 measures applied in relation to the normative group: in Auditory and Visual Attention, Speeded Naming, Comprehension of Instructions, Fingertip Tapping, Imitation Hand Positions, Visuomotor Precision, Design Copy, Arrows, Memory for Faces, Memory for Names and Narrative Memory $(\mathrm{z}$-score $=-.29$ to -1.67 in the $\mathrm{CP}$ group and $\mathrm{z}$-score $=$ .38 to -1.07 in the group at risk). The CP group had lower results in comparison to the group at risk in 5 measures which include a motor component (oral or manual) in performance. The group at risk had worse results in the Memory for Names than the CP group.

Conclusion The children at perinatal risk achieved significantly lower neuropsychological results in comparison to the normative group, regardless of motor deficit (in the most of measures) or any demographic and perinatal variable or EEG findings. It may be concluded that both clinical groups are at risk for specific cognitive deficits. That has significant implications for neuropsychological examination and treatment, where the model of developmental neuropsychological psychotherapy may be applied.

\section{GP229 THE USE OF THE KETOGENIC DIET IN A METABOLIC PATIENT WITH GLYCOGEN STORAGE DISEASE TYPE IIIA}

${ }^{1}$ Christine Merrigan*, Orla Purcell ${ }^{2}$, 'Eimear Forbes, ${ }^{1}$ Jenny Mc Nulty, ${ }^{1}$ Emma Lally, ${ }^{1}$ Prof Ellen Crushell. 'National Centre for Inherited Metabolic Disorders, Dublin, Ireland; ${ }^{2}$ National Centre for Inherited Metabolic Disorders, Dublin, Isle of Man

\subsection{6/archdischild-2019-epa.288}

Background Glycogen Storage Disease (GSD) Type IIIa is a recessively inherited disorder caused by a deficiency in the debranching enzyme amylo-1,6-glucosidase. This deficiency allows for the accumulation of glycogen in the liver, heart and skeletal muscle. Typically patients present in infancy with recurrent hypoglycaemia and hepatomegaly. Standard treatment is a high carbohydrate, moderate to high protein and low fat diet, with regular feeds to help maintain euglycaemia. Conversely the ketogenic diet (KD) consists of a very high fat, low carbohydrate diet with moderate protein intake and has traditionally been used for treatment of epilepsy. It has well noted that ketone bodies can be used as an alternative energy source when carbohydrate intake is low to maintain euglycaemia. 